\title{
Monitoring coupled moisture and salt transport using single vertical suction experiment
}

\author{
Z. Pavlík, J. Mihulka, M. Pavlíková \& R. Černý \\ Department of Materials Engineering and Chemistry, \\ Faculty of Civil Engineering, Czech Technical University in Prague, \\ Czech Republic
}

\begin{abstract}
A new method for simultaneous monitoring of coupled moisture and chloride ions transport is presented in the paper. The experiment is done in the conditions of one-sided 1.0 M NaCl solution vertical uptake into a sample of calcium silicate based material. In the experimental work, rod-shaped sample is used for the determination of moisture and chloride concentration profiles in simulated 1$\mathrm{D}$ water and chloride solution transport. For the measurement, advanced Time Domain Reflectometry (TDR) sensors are used. The sensors allow for moisture content assessment on the basis of relative permittivity measurement and chloride concentration monitoring based on electrical conductivity measurement. On the basis of measured data, moisture and chloride concentration profiles are obtained. Experimentally determined chloride concentration profiles and moisture profiles are then used for identification of apparent chloride diffusion coefficient and moisture diffusivity on the basis of inverse analysis using a simple diffusion model. Finally, the calibration procedure of the applied measuring method is discussed and practical recommendations for application of the combined TDR/electrical conductivity sensors for monitoring of coupled moisture and salt solution transport are given.
\end{abstract}

Keywords: moisture, salt concentration, relative permittivity, electrical conductivity, calcium silicate. 


\section{Introduction}

Moisture and salt induced damage is considered to be one of the essentially problematic elements in the decay of building materials and structures. There is evident that the rising of moisture content in buildings and their materials leads to serious negative events, like degradation of materials (disintegration of inorganic plasters, porous stones and ceramic bricks, binder decomposition, surface erosion, etc.). It has also negative effects on biological devaluation of constructions (mould growing) and on the conditions of interior climate from the point of view of hygienic aspects. Water can deteriorate building materials and structure surfaces by acid decomposition reactions. Typical example is sulphur dioxide that dissolves in water and partly forms sulphurous acid and sulphur trioxide which forms acid as well. Both acids decompose lime and lime-mixed binders in coatings $[1,2]$. The final result is formation of gypsum, $\mathrm{CaSO}_{4} \cdot 2 \mathrm{H}_{2} \mathrm{O}$. The reaction can be expressed in a simplified manner as:

$$
\mathrm{CaCO}_{3}+\mathrm{SO}_{2}+1 / 2 \mathrm{O}_{2}+\mathrm{H}_{2} \mathrm{O} \rightarrow \mathrm{CaSO}_{4} \cdot 2 \mathrm{H}_{2} \mathrm{O}+\mathrm{CO}_{2}
$$

and

$$
\mathrm{CaCO}_{3}+\mathrm{SO}_{3}+2 \mathrm{H}_{2} \mathrm{O} \rightarrow \mathrm{CaSO}_{4} \cdot 2 \mathrm{H}_{2} \mathrm{O}+\mathrm{CO}_{2} .
$$

Gypsum has a large molar volume and, under favourable humidity conditions, large crystals of gypsum form. As a result, crystallisation pressure decays the surface layer of lime based material.

Significant is also the negative effect of moisture on compressive and bending strength of bearing-structures materials. In the areas, where the temperature fluctuates around $0^{\circ} \mathrm{C}$, water gives rise to freeze-thaw weathering that especially deteriorates porous structure of building materials. Ice, compared to the water volume in liquid phase, has a volume that is $9 \%$ higher and its crystallisation pressure causes the damage of solid porous structure of materials.

A part of building damages assigned to the negative moisture effects would not arise, if only pure water would be present. Water represents very often only transport medium for other harmful pollutants that take part in the process of surface degradation of building materials. Water transport in porous materials makes possible salt transport or accumulation as well. Salt accumulation in specific places of building structures can lead in consequence to their failure or destruction. Among water soluble salt action in building materials, especially salt crystallization, salt hydration, hygroscopic water absorption, efflorescence and leaching represent the most harmful effects on the properties of building materials and structures.

Salt crystallisation is physico-chemical degradation process that is related to formation of saturated and oversaturated salt solutions due to water evaporation. After overrun of the range of solubility, the salt crystals are growing and exert crystallisation pressures on walls of porous space. In dependence on the strength of materials, the damage of porous structure of materials is initiated.

Salt hydration is related to salts that are able to bond in their crystal lattice certain defined number of water molecules. They form hydrates what is accompanied by volume changes and hydration pressures. For building materials 
are the most dangerous salts changing their forms at standard climatic conditions; sodium sulphate, sodium carbonate and calcium nitrate. Example of hydration of calcium nitrate is given in equation

$$
\mathrm{Ca}\left(\mathrm{NO}_{3}\right)_{2} \cdot 4 \mathrm{H}_{2} \mathrm{O} \stackrel{30^{\circ} \mathrm{C}}{\longrightarrow} \mathrm{Ca}\left(\mathrm{NO}_{3}\right)_{2} \cdot 3 \mathrm{H}_{2} \mathrm{O} \stackrel{100^{\circ} \mathrm{C}}{\longrightarrow} \mathrm{Ca}\left(\mathrm{NO}_{3}\right)_{2} .
$$

Although the moisture and water soluble salts related problems of buildings and their particular materials are generally known and proven, the exact description of coupled moisture and salt transport mechanism in porous medium remains still open field for building physicists and engineers. On this account, the main motivation of the presented work is to contribute to the explanation of salt solution transport and identification of parameters that can be used for its more exact characterization and description.

\section{Method for simultaneous measurement of moisture and salt concentration}

Water and salt ions possess many anomalous properties, which also affect the properties of a porous material. Therefore, there exist various methods of determination of moisture and salt content in porous materials, and various moisture and salt concentration meters.

The presence of salt ions can negatively affect the accuracy of moisture measurement methods, especially of relative methods, where the measured physical quantity is dependent on salt concentration. Therefore, in case of simultaneous moisture and salt concentration measurement, proper method which accuracy is not influenced by presence of salt ions must be chosen. As stated in literature, the high frequency microwave methods based on permittivity determination correlated to moisture content can be used for such type of measurements.

In this paper we introduce TDR (Time Domain Reflectometry) method for moisture measurement combined with simultaneous assessment of salt concentration by means of electrical conductivity measurement. TDR technique represents specific methodology among the microwave impulse techniques. The principle of TDR device consists in launching of electromagnetic waves and the amplitude measurement of the reflections of waves together with the time intervals between launching the waves and detecting the reflections. Time/velocity of pulse propagation depends on the apparent relative permittivity of the porous material, which can be expressed using the formula

$$
\varepsilon_{r}=\left(\frac{c t_{p}}{2 L_{s}}\right)^{2}
$$

where $\varepsilon_{r}$ is the complex relative permittivity of the porous medium, $c$ the velocity of light $\left(3 \cdot 10^{8} \mathrm{~m} / \mathrm{s}\right), t_{p}$ the time of pulse propagation along the probe rods measured by TDR meter and $L_{\mathrm{s}}$ the length of the sensor's rod inserted into a measured porous medium.

The determination of moisture content using the permittivity measurements is then based on the fact that the static relative permittivity of pure water is equal to 
approximately 80 at $20^{\circ} \mathrm{C}$ [3], while for most dry building materials it ranges from 2 to 6.

For evaluation of moisture content from measured relative permittivity values, three basic approaches can be used. The first possibility is utilization of empirical conversion functions generalized for a certain class of materials. On the basis of analysis performed in [4], it can be stated that empirical conversion functions used in current research for TDR data conversion are anything but universal. They are always limited to specific groups of materials. The second is application of dielectric mixing models, which assumes knowledge of the relative permittivities of the material matrix, water, air and other parameters, that cannot be measured directly but have to be determined by empirical calibration of the model. Dielectric mixing models were tested in many practical applications and their perspectives for further use seem to be better than those of the empirical conversion functions [5-7]. The third method for evaluation of moisture content from measured relative permittivity consists in empirical calibration for the particular material using a reference method, such as the gravimetric method. This method is the most reliable until now and was used also in this work for calibration of TDR method for moisture measurement in calcium silicate based material.

As it was mentioned above, measurement of salt concentration (in our case of chlorides) was done by means of electrical conductivity measurement that is in clear relation with salt concentration. The calibration was done using measurement of salt concentration by ion selective electrode together with measurement of electrical conductivity. In this way, the salt-concentration dependent electrical conductivity was accessed.

\section{Experimental}

The experiment for determination of moisture and salt concentration profiles was done in the conditions of one-sided $1.0 \mathrm{M} \mathrm{NaCl}$ solution vertical uptake into the sample of calcium silicate based material. In the experimental work, rod-shaped sample was used for the determination of moisture and chloride concentration profiles in simulated 1-D water and chloride solution transport. The sample size was $50 / 100 / 300 \mathrm{~mm}$ and all the lateral sides of the sample were vapor proof insulated by epoxy resin to ensure 1-D moisture and salt solution transport. Into the studied sample, 8 two-rod miniprobes LP/ms (Easy Test) were placed for the monitoring of complex relative permittivity and electrical conductivity. The sensors are made of two $53 \mathrm{~mm}$ long parallel stainless steel rods, having $0.8 \mathrm{~mm}$ in diameter and separated by $5 \mathrm{~mm}$ [3]. The sphere of sensor's influence creates the cylinder having diameter about $7 \mathrm{~mm}$ and height about $60 \mathrm{~mm}$, circumference around the rods of sensor. The accuracy of relative permittivity and electrical conductivity reading, and the measuring range of applied sensors are given in Table 1.

For the TDR measurements in this paper, the cable tester TDR/MUX/mts produced by Easy Test which is based on the TDR technology with $\sin ^{2}$-like 
Table 1: $\quad$ Accuracy and measuring range of applied TDR sensors.

\begin{tabular}{|c|c|c|}
\hline Measured quantity & Measuring range & Accuracy \\
\hline Relative permittivity $\varepsilon$ & $2 \div 90$ & $\begin{array}{c}\text { Absolute error: } \\
\pm 1 \text { for } 2 \leq \varepsilon \leq 6 \\
\pm 2 \text { for } \varepsilon \geq 6\end{array}$ \\
\hline Electrical conductivity $\sigma$ & $0 \div 1 \mathrm{~S} / \mathrm{m}$ & $\begin{array}{c}\text { Relative error: } \\
\pm 5 \%\end{array}$ \\
\hline
\end{tabular}

needle pulse having rise-time of about $250 \mathrm{ps,} \mathrm{was} \mathrm{used.} \mathrm{The} \mathrm{working} \mathrm{frequency}$ of this device is $1.8 \mathrm{GHz}$ for the relative permittivity measurement.

The measuring technology was as follows. At first, the sensors were placed into the sample and sealed by silicone gel. Since the material is rather soft, the sensors were placed into the sample by simple impress. Then, the sample was put into a vessel containing $1.0 \mathrm{M} \mathrm{NaCl}$ water solution and the suction has started. The complex relative permittivity and electrical conductivity were continuously monitored and stored in computer. After specific time interval, the experiment was interrupted and the sample was cut to eight separate pieces containing particular sensors. Finally, the sensors were removed and in each piece the moisture content and chloride concentration were measured by reference method. The moisture content was accessed by gravimetric method and chloride concentration by ion selective electrode (device $\mathrm{pH} / \mathrm{ION} 340 \mathrm{i}$ ) applied on leaches from particular sample pieces. In this way, the empirical calibration curves of TDR method for calcium silicate were determined.

The suction experiment was realized on calcium silicate material. It is material having high thermal insulation properties, high total open porosity $(87 \%)$, low bulk density $\left(230 \mathrm{~kg} / \mathrm{m}^{3}\right)$ and from the chemical point of view is formed by $\mathrm{Ca}_{2} \mathrm{SiO}_{4}$. Sample arrangement and measuring technology is visible from Fig. 1.

\section{Determination of moisture diffusivity and chloride diffusion coefficient}

Moisture diffusivity and chloride diffusion coefficient represent necessary input data for computational modeling of coupled moisture and chloride ions transport in porous building materials. Their knowledge is important also for evaluation of moisture and salt solution transport properties of specific materials and plays important role in the process of design of building structures.

In this paper, inverse analysis of experimentally measured moisture and chloride concentration profiles was used for the computational identification of moisture diffusivity and chloride diffusion coefficient of calcium silicate. Inverse analysis is based on the assumed mode of salt solution transport. In the presented work we have assumed only diffusion mechanism of moisture as well as of chloride ions transport. In this way, the chloride diffusion coefficient is considered to be apparent parameter that includes also the effect of chloride ions binding on porous space walls, advection of salt ions, surface diffusion and 
osmotic effects. Compared to simple Fick's diffusion, the dependence of moisture diffusivity on moisture content $\kappa(w)$ and apparent diffusion coefficient on salt concentration $D(C)$ is considered. In this case, the salt mass balance is expressed by equation

$$
\frac{\partial C}{\partial t}=\operatorname{div}(D(C) \operatorname{grad} C),
$$

where $C\left[\mathrm{~kg} / \mathrm{m}^{3}\right]$ is salt concentration in $\mathrm{kg}$ per volume of the dry porous body, $D$ $\left[\mathrm{m}^{2} / \mathrm{s}\right]$ the apparent salt diffusion coefficient. In this way the salt solution transport is formally described by the same parabolic equation with the same boundary and initial conditions usually used for description of water transport. Therefore, the calculation of concentration-dependent diffusion coefficients from the measured salt concentration profiles could be done using basically the same inverse methods as those for the determination of moisture-dependent moisture diffusivity or temperature-dependent thermal conductivity. In this paper, this type of model was employed for determination of both $D(C)$ and $\kappa(w)$ functions. In calculations, we assume that the concentration field $C(x, t)$ and moisture content field $w(x, t)$ are known from the experimental measurements as well as the initial and boundary conditions of the experiment. Using Matano method [8] and applying two Boltzmann transformations we arrive to the following final formulas for calculation of apparent salt (in our case chloride) diffusion coefficient

$$
D\left(C_{0}\right)=\frac{1}{2 t_{0}\left(\frac{d C}{d z}\right)_{z=z_{0}}} \int_{z_{0}}^{\infty} z \frac{d C}{d z} d z,
$$

and moisture diffusivity

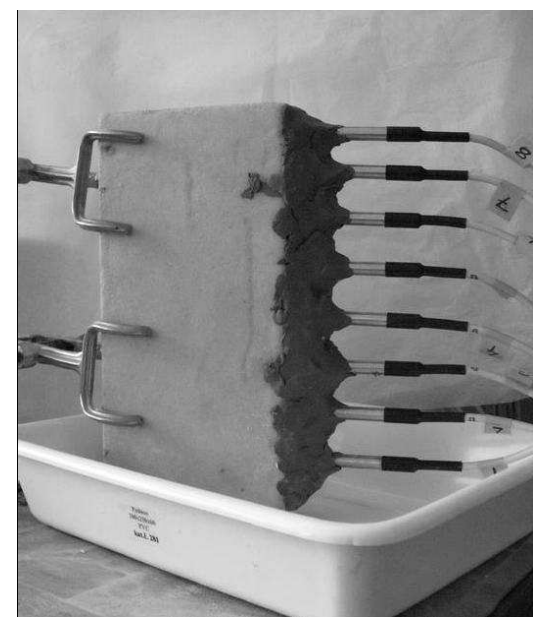

Figure 1: Experimental setup of vertical suction experiment. 


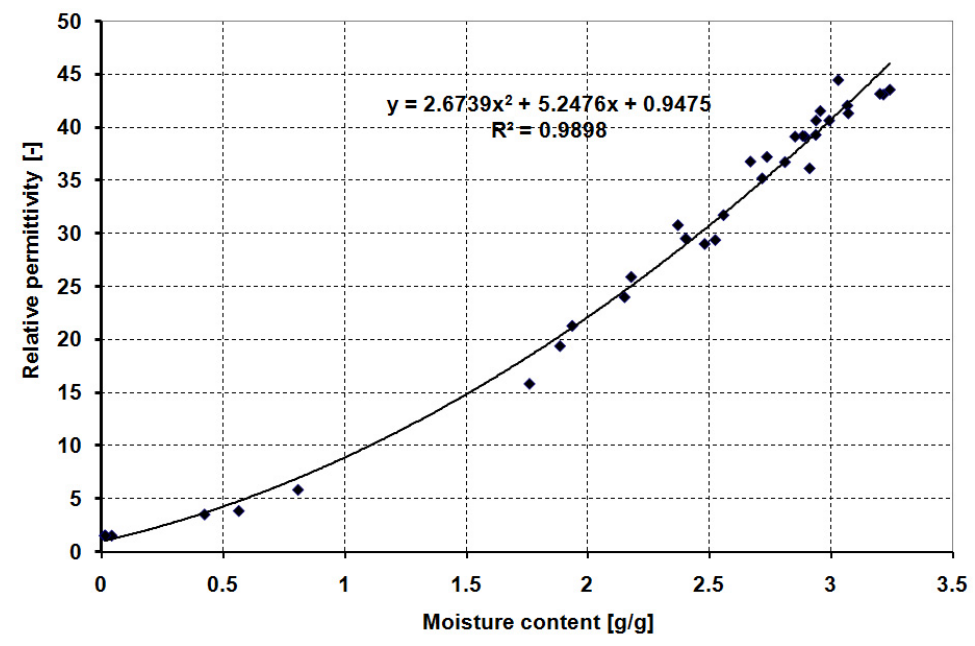

Figure 2: $\quad$ Relative permittivity as function of moisture content.

$$
\kappa\left(w_{0}\right)=\frac{1}{2 t_{0}\left(\frac{d w}{d z}\right)_{z=z_{0}}} \int_{z_{0}}^{\infty} z \frac{d w}{d z} d z,
$$

where $C_{0}=C\left(z_{0}, t_{0}\right)$ is salt concentration in the position $z_{0}$ and time $t_{0}, w_{0}=w\left(z_{0}\right.$, $\left.t_{0}\right)$ the corresponding moisture content and $z$ space variable. The integral in equations (6) and (7) is solved by common numerical methods, such as Simpson's rule. The details on inverse analysis procedure can be found e.g. in [9].

\section{Experimental and computational results}

Figs. 2, 3 present calibration curves of applied measuring techniques for moisture and chloride concentration measurement.

The measured data show the dependences of relative permittivity on moisture content and electrical conductivity on chloride concentration which knowledge is imperative for the applicability of used methods for salt solution transport monitoring. For calibration purposes, the experimental data were smoothed by simple polynomial relations that can be considered as empirical calibration curves of TDR for measurement of chloride water solution transport in calcium silicate. The experimentally measured moisture and chloride concentration profiles are presented in Figs. 4, 5. The data gives clear evidence about the velocity of moisture and chloride ions propagation in calcium silicate material.

Fig. 6 shows the moisture diffusivity as a function of moisture content, Fig. 7 the apparent chloride diffusion coefficient as a function of chloride concentration. These figures reveal the necessity to consider in balance equations the dependence of transport parameters on moisture and salt concentration. 


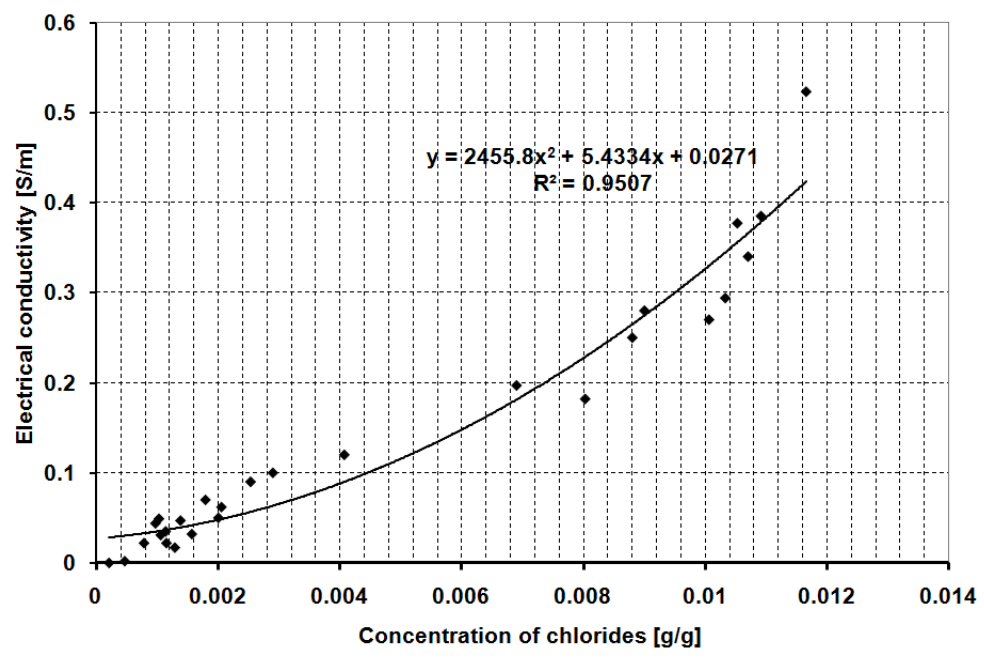

Figure 3: Dependence of measured electrical conductivity on chloride concentration.

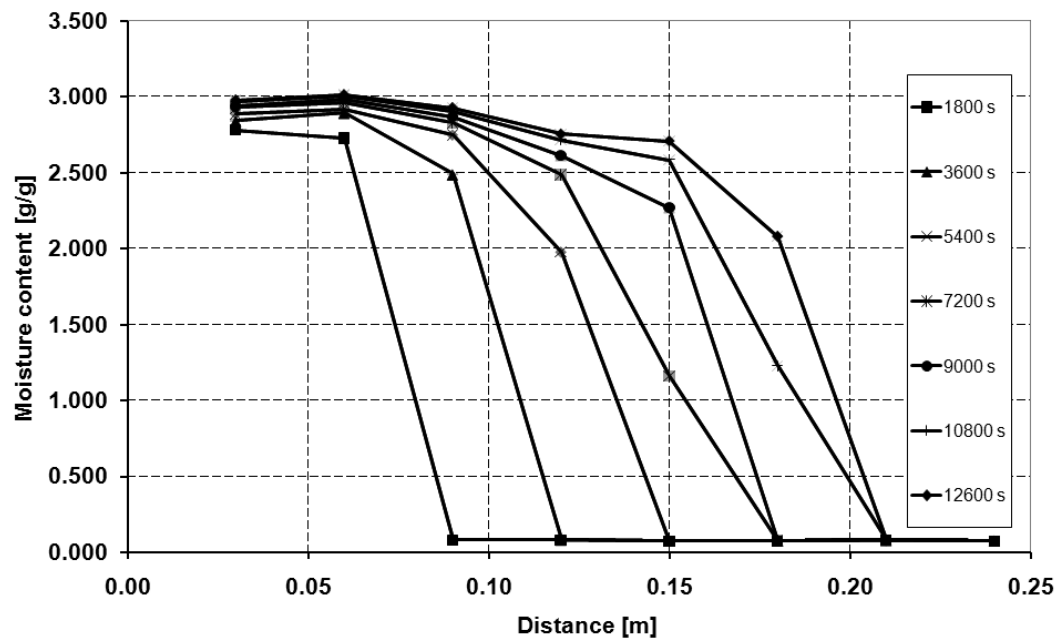

Figure 4: $\quad$ Moisture profiles for calcium silicate measured by TDR.

Looking at the data presented in Fig. 6, the moisture diffusivity varies within the range of four orders of magnitude. A similar trend was found also for concentration dependent chloride diffusion coefficient. In the range of lower concentrations, typically up to $0.005[\mathrm{~g} / \mathrm{g}]$, the apparent diffusion coefficient is close to diffusion coefficient of chlorides in water. On the other hand, in range of higher concentrations it increases very rapidly. 


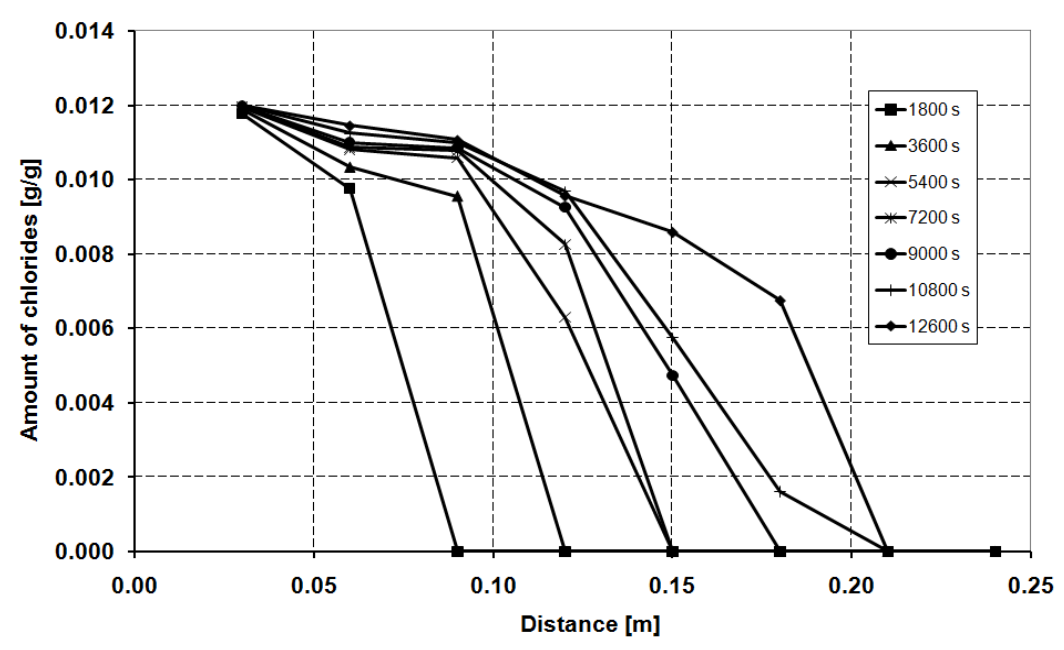

Figure 5: Chloride concentration profiles measured by TDR.

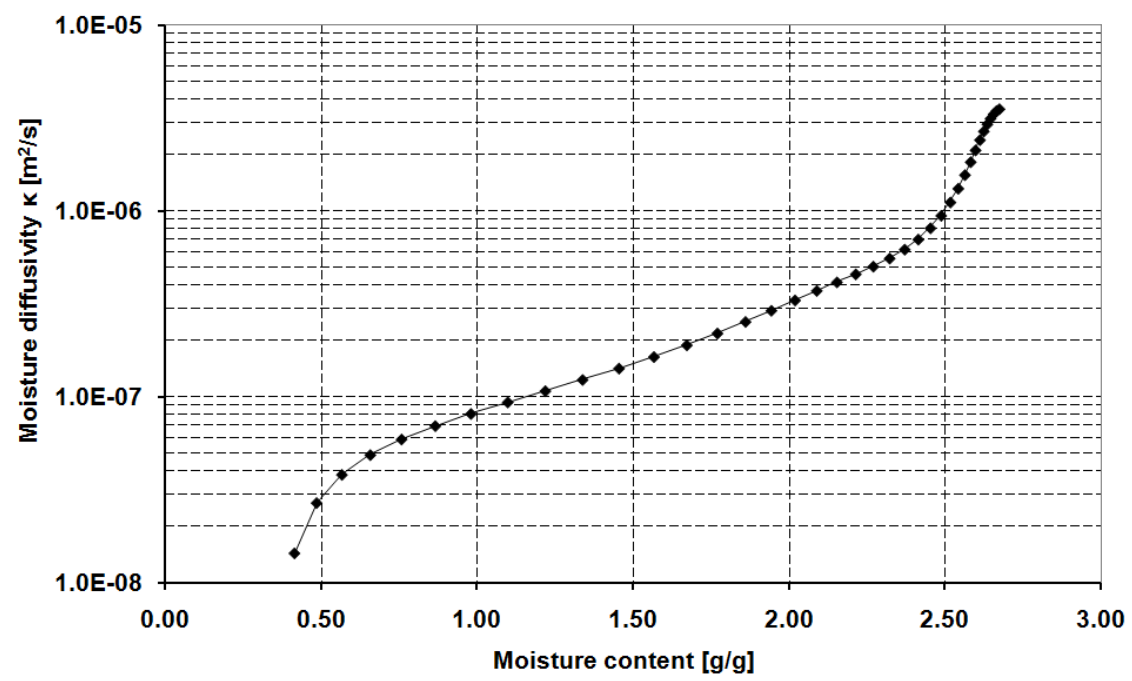

Figure 6: Moisture diffusivity of calcium silicate 


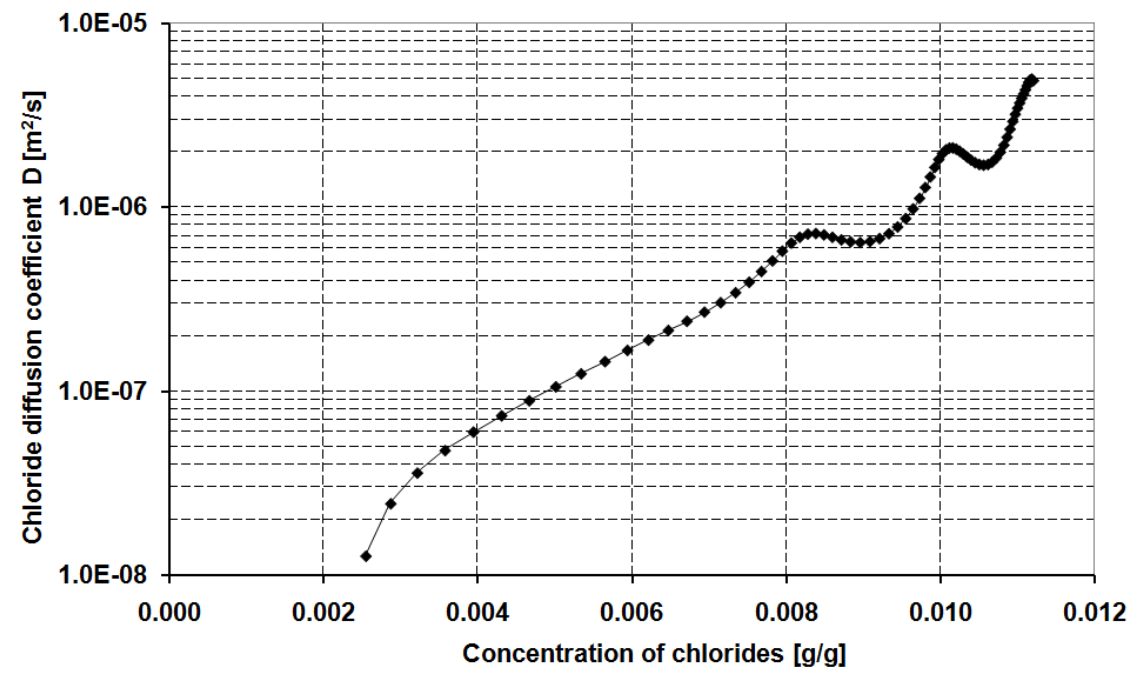

Figure 7: Apparent chloride diffusion coefficient of calcium silicate.

\section{Conclusions}

The experiment presented in this paper has proven the capability of TDR combined sensors for simultaneous monitoring of moisture and salt concentration in porous building materials. This finding is very perspective for future work, especially for building practice that requires complex, precise and reliable methods for moisture and salinity measurement. The assessment of chloride diffusion coefficient and moisture diffusivity represents important information for application of studied calcium silicate material in practice regarding to its intended use in interior thermal insulation systems of building envelopes. This data can also find use in computational modeling of moisture and chloride ions transport in calcium silicate based materials what can be useful for example at damage assessment by means of salt action.

\section{Acknowledgement}

This research has been supported by the Czech Ministry of Education, Youth and Sports, under project No MSM 6840770031.

\section{References}

[1] Rovnaníková, P., Environmental pollution effects on other building materials (Chapter 7). Environmental Deterioration of Materials, ed. A. Moncmanová, WIT Press, Southampton, pp. 217-247, 2007. 
[2] Moncmanová, A., Environmental factors that influence the deterioration of materials (Chapter 1). Environmental Deterioration of Materials, ed. A. Moncmanová, WIT Press, Southampton, pp. 1-21, 2007.

[3] Malicki, M. \& Skierucha, W.M., A Manually Controlled TDR Soil Moisture Meter Operating with 300 ps Rise-Time Needle Pulse. Irrigation Science, Vol. 10, pp. 153-163, 1989.

[4] Fiala, L., Pavlík, Z., Jiřičková, M., Černý, R., Sobczuk, H. \& Suchorab, Z., Measuring Moisture Content in Cellular Concrete Using The Time Domain Reflectometry Method. CD-ROM Proceedings of 5th International Symposium on Humidity and Moisture, J. Brionizio, P. Huang (eds.), Inmetro, Rio de Janeiro, paper No. 103, 2006.

[5] Dobson, M.C., Ulaby, F.T., Hallikainen, M.T. \& El-Rayes, M.A., Microwave dielectric behavior of wet soil, Part II: Dielectric mixing models, IEEE Trans. Geosci. Remote Sensing GE-23, pp. 35-46, 1985.

[6] Jacobsen, O.H. \& Schjonning, P., Comparison of TDR Calibration Functions for Soil Water Determination. Proceedings of the Symposium Time-Domain Reflectometry - Applications in Soil Science, L. W. Petersen and O. H. Jacobsen (eds.), Danish Institute of Plant and Soil Science, Lyngby, pp. 25-33, 1995.

[7] Pavlík, Z., Fiala, L., Pavlíková, M., Černý, R., Sobczuk, H. \& Suchorab, Z., Calibration of the Time Domain Reflectometry Method for Measuring Moisture Content in AAC of Various Bulk Densities, ISEMA 2007, Hamamatsu: Shizuoka University, pp. 151-158, 2007.

[8] Matano, C., On the relation between the diffusion coefficient and concentration of solid metals. Jap. J. Phys., 8, pp. 109-115, 1933.

[9] Fiala, L., Pavlík, Z., Pavlíková, M. \& Černý, R., Water and Chloride Transport Properties of Materials of Historical Buildings. Recent Developments in Structural Engineering, Mechanics and Computation, Rotterdam: Millpress Science Publishers, pp 581-582, 2007. 